\title{
Consciência fonológica no início da escolarização e o desempenho ulterior em leitura e escrita: estudo correlacional
}

\author{
Magda Solange Vanzo Pestun \\ Universidade Estadual de Londrina
}

\begin{abstract}
Resumo
Pesquisas contemporâneas têm demonstrado que habilidades metalingüísticas são fundamentais para aquisição e desenvolvimento da leitura e escrita. Com o propósito de verificar se crianças que não haviam freqüentado a escola até sua inserção no pré III e que não possuíam conhecimento de leitura e soletração apresentavam consciência fonológica ao ingressarem no ensino formal, e se a presença dessa habilidade favorecia a aquisição da leitura e escrita, 167 crianças de ambos os sexos, com idade média de 5 anos e 8 meses, de nível sócio-econômico semelhante, participaram deste estudo, que foi realizado em três etapas. Na primeira etapa (início do pré III), a habilidade de consciência fonológica foi avaliada por meio da Prova de Consciência Fonológica. Nas segunda e terceira etapas (início e término do $1^{\underline{0}}$ ano), as crianças foram reavaliadas em consciência fonológica e avaliadas em leitura oral e escrita sob ditado de palavras e de pseudopalavras. Os resultados indicaram correlação positiva entre consciência fonológica e ulterior desempenho em leitura e em escrita.
\end{abstract}

Palavras-chave: consciência fonológica; leitura; escrita; desenvolvimento

\begin{abstract}
Phonological awareness in the beginning of schooling and ulterior performance in reading and writing: a correlational study. Contemporary research has demonstrated that metalinguistic skills are fundamental for the acquisition and development of reading and writing skills. With the purpose of verifying if children who had not attended school before preschool III, with no knowledge of reading and spelling, presented phonological awareness when entering formal schooling and if the presence of this skill favored reading and writing acquisition, 167 children from both sexes with an average age of 5 years and 8 months old, similar socialeconomical level, took part in this study which was carried out in three stages. In the first stage (beginning of preschool III), the phonological awareness skill was evaluated by means of the Phonological Awareness Test. In the second and third stages (beginning and ending of the first grade of the elementary school), the children were reassessed in their phonological awareness and evaluated in oral reading and writing with dictation of words and pseudowords. The results showed a positive correlation between phonological awareness and ulterior performance in reading as well as in writing.
\end{abstract}

Keywords: phonological awareness; reading; writing; development

$\mathrm{P}$ esquisas contemporâneas têm demonstrado consistentemente que habilidades metalingüísticas, ou seja, a capacidade de refletir sobre a própria língua, são de fundamental importância para a aquisição e o desenvolvimento da leitura e da escrita (Ball \& Blachman, 1991; Bradley \& Bryant, 1983; Cardoso-Martins, 1995).

Uma das habilidades metalingüísticas é a consciência fonológica, isto é, a consciência de que a fala pode ser segmentada e que os segmentos (palavras, sílabas, fonemas) podem ser manipulados. Essa habilidade é desenvolvida gradualmente conforme a criança experimenta situações lúdicas (cantigas de roda, jogos de rima, identificação de sons inici- ais de palavras) e é instruída formalmente em atividades grafofonêmicas. Estudos com crianças não alfabetizadas vêm demonstrando que elas já apresentam, durante a educação infantil, algumas habilidades metafonológicas que contribuem para o sucesso da aprendizagem da leitura e da escrita (Bradley \& Bryant, 1983; Capovilla \& Capovilla, 1998; Carraher \& Rego, 1984; Coimbra, 1997). No entanto, alguns pesquisadores sustentam que as crianças, antes de serem alfabetizadas, não têm uma compreensão clara de como a fala é organizada (Goswani \& Bryant, 1990; Read, Zhang, Nie, \& Ding, 1986). Somente a partir do conhecimento das características da escrita, é que as crianças desenvolveriam a consciência 
fonológica, ou seja, a introdução formal no sistema alfabético seria o fator causal para o desenvolvimento de habilidades metalingüísticas. Recentemente, alguns pesquisadores defendem que, na realidade, existe uma relação de reciprocidade, isto é, ao mesmo tempo em que as habilidades metalingüísticas são fundamentais para a aquisição e o desenvolvimento da leitura e da escrita, o treinamento em leitura favorece o desenvolvimento da consciência fonológica (Adams, 1990; Morais, Mousty, \& Kolonsky, 1998).

Independentemente de a relação entre consciência fonológica e habilidade para leitura e escrita ser de causa, de efeito ou de reciprocidade, o que se tem claro atualmente é que o domínio fonológico exerce grande influência no processo de aprendizagem da leitura e da escrita, uma vez que possibilita a generalização dos sistemas de escrita alfabéticos.

se o alfabeto é um código com sistema de regras que asseguram a cada fonema de uma língua sua representação gráfica distinta, estas regras permitem compreender uma série de signos ortográficos nunca vistos anteriormente e permitem criar novas séries de signos para representar conceitos novos, de maneira que suas representações sejam inteligíveis para toda pessoa que conheça o sistema. (Batista \& Resende da Costa, 2003, p. 41)

Segundo Capovilla e Capovilla (2003), diversos países de língua alfabética compreenderam rapidamente que, para evitar dificuldades em leitura e escrita, as crianças deveriam ser ensinadas, de forma explícita e sistemática, a manipular fonemas por meio da instrução. Quanto mais desenvolvida essa habilidade, melhor a compreensão da relação fonema-grafema.

No Brasil, alguns pesquisadores vêm verificando as habilidades metalingüísticas em crianças e construindo instrumentos para avaliar a consciência fonológica e a futura habilidade de leitura e escrita, bem como propondo programas de treinamento a serem implementados desde a Educação Infantil (Capovilla \& Capovilla, 1998, 2000; Cardoso-Martins, 1995; Moojen et al., 2003).

Como no Paraná as pesquisas que verificam as habilidades metalingüísticas são ainda incipientes, resolveu-se desenvolver esta pesquisa com o propósito de verificar se crianças pré-escolares, sem nenhum conhecimento de leitura e soletração, apresentavam consciência fonológica ao ingressarem no ensino formal e se a presença dessa habilidade favorecia a aquisição de leitura e escrita.

\section{Método}

Este estudo longitudinal de caráter experimental foi iniciado em fevereiro de 2002 com 167 crianças e concluído em novembro de 2003 com 132 crianças ( 75 do sexo feminino e 57 do sexo masculino). Trinta e cinco alunos solicitaram transferência de escola ao longo dos dois anos da pesquisa. A idade média no início do estudo era de cinco anos e oito meses (mínima de cinco anos e máxima de seis anos e seis meses). As crianças freqüentavam o pré III em três escolas públicas municipais pertencentes ao Jardim Santo Amaro, município de Cambé, PR, e apresentavam nível sócio-econômico semelhante (renda média familiar mensal de dois salários mínimos).
Todos os sujeitos tinham o consentimento de seus pais ou responsáveis para participarem da pesquisa e, é importante ressaltar, nenhuma dessas crianças havia freqüentado anteriormente o ensino formal, portanto, não liam nem soletravam ao início do estudo.

Todas as fases de coleta de dados foram realizadas nas três escolas participantes, em horário normal de aulas, em salas reservadas para atendimentos individuais ou de pequenos grupos.

Foram empregados como instrumentos de avaliação:

(1) Roteiro de Anamnese para a entrevista com os pais ou responsáveis pela criança;

(2) Prova de Consciência Fonológica (PCF, ver Capovilla \& Capovilla, 1998), que tem por objetivo avaliar a habilidade de crianças de manipular os sons da fala. Consiste em 40 questões de teste e duas de treino, divididas em 10 blocos de 4 perguntas cada. Os blocos avaliam habilidades de síntese silábica e fonêmica, análise silábica e fonêmica, rima, aliteração, segmentação silábica e fonêmica e transposição silábica e fonêmica. Os resultados são apresentados como escore ou freqüência de acertos, sendo 40 acertos o máximo possível; (3) Escala Colúmbia de Maturidade Intelectual (ECMI, Burgemeister, Blum, \& Lorge, 1971, adaptação de Rodrigues \& Pio da Rocha, 1994). Mede a aptidão geral de raciocínio de crianças na faixa etária de 3 anos e 6 meses a 9 anos e 11 meses. A medida escolhida pela pesquisadora para efeito de análise foi o estanino, um escore padronizado que varia de 1 a 9 pontos. O estanino é obtido a partir do número de acertos da criança no teste e de sua idade cronológica;

(4) lista de palavras familiares e de pseudopalavras (Pinheiro, 1994) para leitura oral e tomada de ditado. Composta de 192 itens psicolingüísticos, que variam em termos de lexicalidade, regularidade, freqüência e comprimento. Dos 192 itens, 96 são palavras familiares e 96 são pseudopalavras. Das 96 palavras familiares, 32 são regulares, 32 irregulares e 32 regra, sendo 48 de alta freqüência e 48 de baixa freqüência. Tanto as palavras familiares quanto as pseudopalavras são divididas em dissílabas e trissílabas;

(5) gravadores (para gravar as respostas nas provas PCF);

(6) fitas-cassete;

(7) cronômetros;

(8) folhas de respostas para leitura oral e tomada de ditado de palavras familiares e pseudopalavras.

\section{Procedimento}

Este estudo longitudinal, de caráter experimental, foi realizado em três etapas:

Etapa I: quando as crianças iniciaram o pré III (fevereiro e março de 2002);

Etapa II: quando as crianças iniciaram o $1^{\underline{0}}$ ano do ensino fundamental (fevereiro e março de 2003);

Etapa III: quando as crianças concluíram o $1^{\underline{0}}$ ano do ensino fundamental (dezembro de 2003).

Na Etapa I, os pais ou responsáveis pelas crianças participantes foram convidados para uma entrevista inicial, elaborada sob a forma de um questionário (roteiro de anamnese), cujo objetivo era obter o máximo de informações possível 
com relação ao desenvolvimento neuropsicomotor, sócio afetivo e escolar de seu filho ou filha, bem como alguma intercorrência que pudesse ter havido durante os períodos pré, peri e pós-natal. A idade em que a criança falou as primeiras palavras com significado, seu desenvolvimento lingüístico, psicomotor e possíveis casos de dificuldade escolar na família eram considerados dados significativos na história do sujeito. Na ocasião, os objetivos do trabalho foram explicitados pela pesquisadora, a qual solicitou aos pais ou responsáveis a anuência para a participação de seu filho(a) na pesquisa, mediante assinatura do Termo de Consentimento Livre e Esclarecido. Na mesma ocasião, as crianças foram submetidas à avaliação individual do potencial intelectual, por meio da ECMI, e da consciência fonológica, por meio da PCF (F0). Cada prova foi aplicada em uma sessão de 30 minutos, com exceção da entrevista de anamnese, cuja duração foi de 60 minutos.

Na Etapa II, as crianças matriculadas na $1^{\mathrm{a}}$ série foram submetidas novamente à avaliação da consciência fonológica, por meio da PCF (F1), de leitura oral de 48 palavras familiares (L1) e 48 pseudopalavras (LP1), e de escrita sob ditado de outras 48 palavras familiares (D1) e 48 pseudopalavras (DP1). $\mathrm{O}$ dado de interesse para a análise foi a freqüência de acertos para cada modalidade avaliada (palavras familiares e pseudopalavras). As provas de consciência fonológica e leitura oral de palavras familiares e de pseudopalavras foram realizadas em duas sessões individuais de 30 minutos cada. As crianças eram retiradas da sala de aula e conduzidas pela pesquisadora à sala de avaliação. Todas as respostas foram gravadas, para futura pontuação e análise. Após o término da avaliação, elas eram reconduzidas à sala de aula. A Prova de Tomada de Ditado foi realizada em três sessões de 30 minutos cada.

Na Etapa III, as crianças foram novamente avaliadas em consciência fonológica por meio da PCF (F2), em leitura de palavras familiares (L2) e de pseudopalavras (LP2) e em tomada de ditado de palavras familiares (D2) e de pseudopalavras (DP2). O procedimento foi semelhante ao da Etapa II.

A análise dos resultados das provas realizadas ao longo dos dois anos de estudo foi conduzida, primeiramente, considerando-se a média aritmética obtida em todas as variáveis medidas (consciência fonológica - F0, F1, F2; leitura de palavras familiares - L1 e L2; leitura de pseudopalavras - LP1 e LP2; ditado de palavras familiares - D1 e D2 e ditado de pseudopalavras - DP1 e DP2), somente das crianças que participaram das três etapas da pesquisa $(n=132)$.

Analisando as estatísticas de tendência central, observou-se que a mediana ou era nula ou era muito baixa para a maioria das variáveis. Optamos, portanto, pela média aritmética como valor de referência, pois a mesma permitiria melhor avaliação com os demais momentos. Analisamos as mudanças ocorridas ao longo dos dois anos de pesquisa em relação à média de referência (considerada como as médias das primeiras medidas: F0, L1, LP1, D1, DP1), por meio do teste de Quiquadrado (McNemar), com o objetivo de analisar se as mudanças foram para melhor ou para pior. A comparação dos grupos acima e abaixo da média de referência (F0) em todos os momen- tos foi realizada com o teste de Mann-Whitney. Os testes estatísticos foram realizados em nível de significância de 5\% e processados no programa SAS (Statistical Analysis System).

\section{Resultados}

Os dados das entrevistas realizadas com os pais ou responsáveis pelas crianças não revelaram informações significativas (deficiências visuais ou auditivas, alterações neurológicas importantes, como paralisia cerebral, epilepsia) que impedissem a participação da criança na pesquisa. Na ECMI, o escore médio foi de 4,16, sendo o escore mínimo de 1 e o escore máximo de 8.

Houve um crescimento significativo nos desempenhos dos alunos em consciência fonológica, leitura e escrita sob ditado de palavras e de pseudopalavras, da primeira avaliação realizada no início do pré-III à última avaliação realizada ao término do primeiro ano do ensino fundamental (Tabela 1).

Tabela 1

Estatísticas descritivas das variáveis mensuradas

\begin{tabular}{lrrrrr}
\hline Variáveis & Média & $\begin{array}{c}\text { Desvio } \\
\text { padrão }\end{array}$ & $\begin{array}{c}\text { Valor } \\
\text { Mínimo }\end{array}$ & Mediana & $\begin{array}{c}\text { Valor } \\
\text { Máximo }\end{array}$ \\
\hline F0 & 11,3 & 4,3 & 0 & 11,0 & 28 \\
F1 & 18,7 & 6,2 & 0 & 18,5 & 32 \\
F2 & 27,1 & 7,4 & 7 & 28,8 & 40 \\
L1 & 7,5 & 12,3 & 0 & 1,0 & 45 \\
L2 & 34,1 & 14,7 & 0 & 41,0 & 47 \\
LP1 & 6,0 & 10,5 & 0 & 0,0 & 45 \\
LP2 & 28,8 & 13,5 & 0 & 33,0 & 46 \\
D1 & 2,9 & 5,4 & 0 & 0,0 & 30 \\
D2 & 19,8 & 9,5 & 0 & 21,0 & 43 \\
DP1 & 3,9 & 6,7 & 0 & 0,0 & 35 \\
DP2 & 16,2 & 8,7 & 0 & 17,0 & 37 \\
\hline
\end{tabular}

Nota: 0 = fevereiro e março de 2002

$1=$ fevereiro e março de 2003

2 = dezembro de 2003

$\mathrm{F}=$ Prova de Consciência Fonológica (PCF)

$\mathrm{L}=$ leitura oral de 48 palavras familiares

LP = leitura oral de 48 pseudopalavras

$\mathrm{D}=$ escrita sob ditado de outras 48 palavras familiares

DP = escrita sob ditado de 48 pseudopalavras

Quanto à PCF, na primeira avaliação os melhores desempenhos foram observados nos subtestes síntese silábica, segmentação silábica, rima e aliteração. Os piores desempenhos foram observados nos subtestes transposição silábica, transposição fonêmica, segmentação fonêmica e manipulação fonêmica. No decorrer dos dois anos de pesquisa as maiores evoluções foram observadas em: manipulação silábica, segmentação silábica, manipulação fonêmica, transposição silábica e aliteração. Maiores detalhes podem ser obtidos em Pestun (2004).

Analisando a média de referência (momento inicial) de todas as variáveis mensuradas, observou-se que houve mudança significativa no desempenho das crianças para categorias melhores determinadas pelos valores acima da média de referência $(p<0,001)$ como pode ser verificado na Tabela 2 . 
Tabela 2

Número e porcentagem de crianças de acordo com as alterações em relação à média de referência das variáveis mensuradas

\begin{tabular}{lrrrrrrrrr}
\hline \multirow{2}{*}{ Medidas comparadas } & \multicolumn{2}{c}{ Ab-Ab } & \multicolumn{2}{c}{ Ab-Ac } & \multicolumn{2}{c}{ Ac-Ab } & \multicolumn{2}{c}{ Ac-Ac } & \multirow{2}{*}{$p^{*}$} \\
& $\mathrm{n}$ & \multicolumn{1}{c}{$\%$} & $\mathrm{n}$ & $\%$ & $\mathrm{n}$ & $\%$ & $\mathrm{n}$ & $\%$ & \\
\hline F0 - F1 & 15 & 11,4 & 59 & 44,7 & 2 & 1,5 & 56 & 42,4 & $<0,001$ \\
F0 - F2 & 3 & 2,3 & 71 & 53,8 & - & - & 58 & 43,9 & $<0,001$ \\
L1 - L2 & 15 & 11,4 & 81 & 61,4 & - & - & 36 & 27,3 & $<0,001$ \\
LP1 - LP2 & 15 & 11,4 & 77 & 58,3 & - & - & 40 & 30,3 & $<0,001$ \\
D1 - D2 & 12 & 9,1 & 81 & 61,4 & - & - & 39 & 29,5 & $<0,001$ \\
DP1 - DP2 & 15 & 11,4 & 76 & 57,6 & 1 & 0,8 & 40 & 30,3 & $<0,001$ \\
\hline
\end{tabular}

Nota: média de referência = média de todas as crianças nas primeiras medidas $\mathrm{Ab}-\mathrm{Ab}=$ abaixo da média de referência no primeiro e segundo momento $\mathrm{Ab}-\mathrm{Ac}=$ abaixo da média de referência no primeiro momento e acima no segundo momento $\mathrm{Ac}-\mathrm{Ab}$ = acima da média de referência no primeiro momento e abaixo no segundo momento Ac-Ac = acima da média de referência no primeiro momento e acima no segundo momento * Teste de qui-quadrado de McNemar com 1 grau de liberdade

A quantidade de crianças que demonstraram desempenhos acima da média em F0, manteve-se em F1 e aumentou em F2 (Ac-Ac). Somente duas crianças, na segunda avaliação da PCF mudaram de categoria, de acima da média para abaixo da média (Ac-Ab). Provavelmente, alguma variável não controlada determinou esse resultado. Interessante observar o aumento significativo de crianças que mudaram de categoria, ou seja, que apresentavam desempenhos abaixo da média em F0 e evoluíram em F1 (Ab-Ac). Somente três crianças mantiveram desempenhos abaixo da média nas três avaliações da PCF (Ab-Ab).

Em leitura de palavras e de pseudopalavras, 15 crianças mantiveram seus desempenhos abaixo da média nas duas medidas obtidas (Ab-Ab), enquanto houve um acréscimo significativo na quantidade de crianças que evoluíram em leitura (Ab-Ac).

Em escrita sob ditado de palavras familiares, 12 crianças mantiveram seus desempenhos abaixo da média nas duas medidas obtidas. Em escrita sob ditado de pseudopalavras, 15 crianças mantiveram seus desempenhos abaixo da média nas duas medidas obtidas (Ab-Ab), enquanto houve um acréscimo significativo na quantidade de crianças que evoluíram em escrita sob ditado (Ab-Ac). Somente uma criança mudou de categoria, de acima da média para abaixo da média em escrita sob ditado de pseudopalavras (Ac-Ab). Provavelmente, alguma variável não controlada determinou esse resultado.

A Tabela 3 demonstra que em todas as variáveis mensuradas, as crianças que apresentaram escores acima da média nas três avaliações da PCF (F0, F1, F2), também apresentaram escores medianos maiores nas segundas medidas obtidas $(p<0,001)$.

\section{Discussão}

A presente pesquisa teve por objetivo verificar se haveria uma relação causal entre a habilidade de consciência fonológica presente antes da escolarização formal e a aquisição e o desenvolvimento da leitura e da escrita ao término do $1^{\underline{0}}$ ano do ensino fundamental. Como pode ser verificado na

Tabela 3

Escores médios, desvio padrão e mediana das variáveis mensuradas de acordo com a classificação acima ou abaixo da média de referência (F0) em todos os momentos (F0, F1 e F2)

\begin{tabular}{|c|c|c|c|c|c|c|c|}
\hline \multirow{3}{*}{ Variáveis } & \multicolumn{6}{|c|}{ Acima da média de referência em todos os momentos } & \multirow{3}{*}{$p^{*}$} \\
\hline & \multicolumn{3}{|c|}{$\operatorname{Sim}(n=56)$} & \multicolumn{3}{|c|}{ Não $(n=76)$} & \\
\hline & Média & Desvio padrão & Mediana & Média & Desvio padrão & Mediana & \\
\hline L 1 & 10,32 & 14,10 & 4,50 & 5,38 & 10,47 & 0,00 & 0,002 \\
\hline L 2 & 41,21 & 8,57 & 42,00 & 28,91 & 16,21 & 35,50 & $<0,001$ \\
\hline LP1 & 8,70 & 12,18 & 3,50 & 4,04 & 8,80 & 0,00 & $<0,001$ \\
\hline LP2 & 35,64 & 8,85 & 38,00 & 23,70 & 14,28 & 27,50 & $<0,001$ \\
\hline D1 & 4,46 & 6,32 & 1,00 & 1,75 & 4,25 & 0,00 & $<0,001$ \\
\hline D2 & 24,04 & 7,65 & 24,00 & 16,71 & 9,59 & 18,00 & $<0,001$ \\
\hline DP1 & 6,05 & 8,20 & 3,00 & 2,32 & 4,96 & 0,00 & $<0,001$ \\
\hline DP2 & 19,36 & 6,60 & 19,00 & 13,84 & 9,34 & 14,50 & $<0,001$ \\
\hline
\end{tabular}

* Teste de Mann-Whitney 
Tabela 3, parece haver uma relação causal entre todas as variáveis, isto é, entre consciência fonológica, leitura oral e escrita de palavras e de pseudopalavras. Esses resultados corroboram o que os estudos longitudinais documentados na literatura nacional e internacional vêm divulgando (CardosoMartins, 1991; Lundberg, Frost, \& Peterson, 1988; Torgesen, Wagner, \& Rashotti, 1994). Crianças que apresentam consciência de que a fala pode ser segmentada e que os segmentos podem ser manipulados adquirem e desenvolvem as habilidades de leitura e escrita de forma mais eficiente.

No presente estudo, $42 \%$ dos alunos apresentaram consciência fonológica ao início do pré III, ou seja, obtiveram resultados acima da média de referência - responderam corretamente a 11 ou mais de 40 questões propostas. Capovilla e Capovilla (1998) aplicaram a mesma prova em 34 crianças de nível sócio-econômico médio, alunos de pré III de uma escola particular do interior de São Paulo, e obtiveram melhores resultados em consciência fonológica, média de acertos de 18,94\% questões em 40 propostas. Algumas hipóteses podem ser levantadas para explicar a diferença nos resultados entre os dois estudos: (1) a falta de escolarização anterior ao pré III nos alunos do presente estudo pode ter sido a responsável por esses dados. Pesquisas têm demonstrado que a instrução formal no sistema alfabético é muito importante para o desenvolvimento de alguns níveis de consciência fonológica considerados mais complexos, como é o caso da análise e síntese fonêmica, transposição fonêmica (Bertelson, Gelder, Tfouni, \& Morais, 1989; Maluf \& Barrera, 1997; Morais, Bertelson, Cary, \& Alégria, 1986; Morais, Content, Cary, Mehler \& Segui, 1989). A amostra de Capovilla e Capovilla (1998) freqüentava escola particular desde o pré-I; (2) nível sócio-econômico como os pais das crianças do presente estudo eram, em sua maioria, semi-analfabetos ou apresentavam poucos anos de escolarização, provavelmente, liam menos para seus filhos e realizavam poucos jogos de linguagem, o que parece não ter favorecido o desenvolvimento da consciência fonológica nesse grupo. A literatura atual vem demonstrando que pais com pouca escolarização lêem menos para seus filhos e fazem poucos jogos de linguagem com eles (Morais, 1995). As crianças da amostra de Capovilla e Capovilla (1998) pertenciam a uma classe sócio-econômica média, portanto, diferente da amostra do presente estudo.

A mudança significativa no desempenho das crianças para categorias melhores determinadas pelos valores acima da média de referência parece indicar que a introdução formal no sistema alfabético favoreceu o desenvolvimento de habilidades metalingüisticas. Segundo Goswani e Bryant (1990) e Read et al. (1986), as crianças antes de serem alfabetizadas, não têm uma compreensão clara de como a fala é organizada. Somente a partir do conhecimento das características da escrita é que as crianças desenvolvem a consciência fonológica.

Por outro lado, pode-se verificar que os melhores alunos em consciência fonológica (F0, F1, F2) eram os melhores em leitura e em escrita em todos os momentos, o que parece confirmar que habilidades metalingüisticas contribuem para o sucesso da aprendizagem da leitura e da escrita (Bradley \&
Bryant, 1983; Capovillla \& Capovilla, 1998; Carraher \& Rego, 1984; Coimbra, 1997).

Portanto, os resultados desta pesquisa parecem indicar uma relação de reciprocidade, ou seja, assim como as habilidades metalingüísticas são importantes para a aquisição e o desenvolvimento da leitura e da escrita, o ensino destas últimas, favorece o desenvolvimento da consciência fonológica.

Ao longo das três avaliações da PCF, as habilidades de consciência fonológica que mais evoluíram foram aquelas que envolviam sílabas, permanecendo como as mais difíceis as atividades que envolviam fonemas. Segundo Morais et al. (1986, 1989) e Bertelson et al (1989), as sílabas são unidades lingüísticas naturalmente isoláveis na pronúncia e o seu domínio desenvolve-se informalmente através de experiências com a linguagem oral, presentes, portanto, desde cedo nas crianças. O fonema, por sua vez, é uma estrutura de maior complexidade que precisa ser ensinado explicitamente. Conforme Maluf e Barrera (1997), as habilidades para segmentar palavras em suas unidades silábicas e/ou fonêmicas aumentam com o aumento da idade e do nível escolar. Essas mesmas autoras nos revelam que as crianças, participantes de sua pesquisa, na faixa etária dos quatro anos, mostraram maior freqüência de segmentação silábica, ao passo que as crianças na faixa etária de seis anos mostraram um predomínio de segmentação fonêmica. Resultados semelhantes foram obtidos por Liberman, Shankweiler, Fisher e Carter (1974). Nenhuma das crianças de quatro anos pesquisadas por eles conseguiu segmentar em fonemas, mas metade delas conseguiu segmentar em sílabas. Aos seis anos, 70\% já conseguiam segmentar em fonemas e $90 \%$ em sílabas. Entre os quatro e os seis anos, essas crianças aprenderam a ler, a soletrar e a escrever. Acreditamos que o desconhecimento, por parte dos professores de nossa pesquisa, do que são habilidades metalingüísticas e metafonológicas e, consequentemente, a ausência de treinamento sistemático dessas habilidades não favoreceu um melhor desempenho das crianças nas tarefas de segmentação, síntese, manipulação e transposição fonêmica, apesar de estarem concluindo a $1^{\underline{a}}$ série e com idade média de oito anos.

Com relação às crianças que não evoluíram ao longo dos dois anos deste estudo, levantamos a hipótese de elas serem portadoras de um distúrbio de linguagem. Estudos controlados com essas crianças estão sendo atualmente conduzidos.

\section{Considerações finais}

As análises dos resultados obtidos na presente pesquisa permitem indicar que: (1) parece existir uma relação causal entre consciência fonológica e ulterior desempenho em leitura e escrita, visto que a consciência fonológica foi medida antes de as crianças terem aprendido a ler e a soletrar; (2) parece existir uma relação de efeito entre o ensino formal no sistema alfabético e o desenvolvimento da consciência fonológica; (3) existem diferentes níveis de consciência fonológica, ou seja, primeiro desenvolve-se a consciência da sílaba e, posteriormente, a consciência do fonema; (4) as ha- 
bilidades metafonológicas são dependentes da idade, do nível escolar e das formas de ensino.

Assim, sugerimos que: (1) a Educação Infantil pode ser o momento adequado para o desenvolvimento de habilidades metafonológicas que favoreceriam ulterior aquisição e desenvolvimento das habilidades de leitura e de escrita; (2) os professores de Educação Infantil e Ensino Fundamental necessitam de cursos de formação continuada para compreenderem o que é Metalinguagem e Habilidades Metafonológicas e aplicar procedimentos que favoreçam a aquisição e o desenvolvimento dessas habilidades em seus alunos; (3) o desempenho em consciência fonológica parece ser um bom indicador de sinais de risco para Distúrbio de Leitura e Escrita.

\section{Agradecimentos}

Nossos sinceros agradecimentos a Profa. Dra. Tieme Matsuo, docente do Departamento de Matemática Aplicada, Universidade Estadual de Londrina, pelo tratamento estatístico dos dados desta pesquisa. Igualmente manifestamos nosso agradecimento ao Fundo de Apoio ao Ensino, Pesquisa e Extensão, da Universidade Estadual de Londrina (FAEPE/ UEL), pelo apoio.

\section{Referências}

Adams, M. J. (1990). Beginning to read: thinking and learning about print. Cambridge, Massachusetts: MIT.

Ball, E., \& Blachman, B. (1991). Does phoneme awareness training in kindergarten make a difference in early word recognition and developmental spelling? Reading Research_Quarterly, 26(1), 49-66.

Batista, A. S., \& Resende da Costa, M. P. (2003). Competência metalinguística e produção escrita de surdos de diferentes abordagens comunicativas e educacionais. In M. C. Marquezini, M. A. Almeida, \& E. D. Tanaka (Org.), Leitura, escrita e comunicação no contexto da educação especial (pp. 3950). Londrina: Editora da Universidade Estadual de Londrina.

Bertelson, P., Gelder, B., Tfouni, L. V., \& Morais, J. (1989). Metaphonological abilities of adult illiterates: new evidence of heterogeneity. European Journal of Cognitive_Psychology, 1, 239-250.

Bradley, L., \& Bryant, P. (1983). Categorising sounds and learning to read: a causal connection. Nature, 301(5899), 419-421.

Burgemeister, B., Blum, L., \& Lorge, I. (1971). Columbia Mental Maturity Scale. Nova York: Harcourt, Brace, \& Jovanovich.

Capovilla, A. G. S., \& Capovilla, F. C. (1998). Treino de consciência fonológica de pré a segunda série: efeitos sobre habilidades fonológicas, leitura e escrita. Temas sobre Desenvolvimento, 7(40), 5-15.
Capovilla, A. G. S., \& Capovilla, F. C. (2000). Problemas de leitura e escrita: como identificar, prevenir e remediar numa abordagem fônica. São Paulo: Memnon.

Capovilla, A. G. S., \& Capovilla, F. C. (2003). Comparando métodos de alfabetização: evidência da superioridade do método fônico em relação ao método global. In M. C. Marquezine, M. A. Almeida, \& E. D. Tanaka (Orgs.), Leitura, escrita e comunicação no contexto da educação especial (pp. 1-28). Londrina: Editora da Universidade Estadual de Londrina.

Cardoso-Martins, C. (1991). Awareness of phonemes and alphabetic literacy acquisition. British Journal of Educational Psychology, 61, 164-173.

Cardoso-Martins, C. (1995). Sensitivity to rhymes, syllables, and phonemes in literacy acquisition in Portuguese. Reading Research Quarterly, 30(4), 808-827.

Carraher, T. N., \& Rego, L. L. B. (1984). Desenvolvimento cognitivo e alfabetização. Revista Brasileira de Estudos Pedagógicos, 65, 38-55.

Coimbra, M. (1997). Metaphonological ability to judge phonetic and phonological acceptability in five-year-old monolingual and bilingual children. Tese de Doutorado não-publicada, Pontifícia Universidade Católica do Rio Grande do Sul, Porto Alegre.

Goswani, U., \& Bryant, P. (1990). Phonological skills and learning to read. Hove, Reino Unido: Erlbaum.

Liberman, I. Y., Shankweiler, D., Fisher, E., \& Carter, B. (1974). Explicit syllable and phoneme segmentation in the young child. Journal of Experimental Child Psychology, 18, 201-212.

Lundberg, I., Frost, J., \& Petersen, O. (1988). Effects of an extensive program for stimulating phonological awareness in preschool children. Reading Research Quarterly, 23, 262-284.

Maluf, M. R., \& Barrera, S. D. (1997). Consciência fonológica e linguagem escrita em pré-escolares. Psicologia:Reflexão e Crítica, 10(1), 125-145.

Moojen, S., Lamprecht, R., Santos, R. M., Freitas, G. M., Brodacz, R., Siqueira, M., Costa, A. C., \& Guarda, E. (2003). CONFIAS Consciência Fonológica: Instrumento de Avaliação Seqüencial_São Paulo: Casa do Psicólogo.

Morais, J., Bertelson, P., Cary, L., \& Alegria, J. (1986). Literacy training and speech segmentation. Cognition, 24, 45-64.

Morais, J., Content, A., Cary, L., Mehler, J., \& Segui, J. (1989). Syllabic segmentation and literacy. Language and Cognitive Processes, 4, 57-67.

Morais, J., Mousty, P., \& Kolinsky, R. (1998). Why and how phoneme awareness helps learning to read. In C. Hulme \& R. M. Joshi (Orgs.), Reading and spelling: development and disorders (pp. 127-151). New Jersey: Erlbaum.

Morais, J. (1995). A arte de ler. São Paulo: Editora UNESP.

Pestun, M. S. V. (2004). Investigação precoce da consciência fonológica e futura habilidade de leitura e escrita. Temas sobre Desenvolvimento, 13(73), 5-12.

Pinheiro, A. M. V. (1994). Leitura e escrita: uma abordagem cognitiva. Campinas: Psy II.

Read, C., Zhang, Y., Nie, H., \& Ding, B. (1986). The ability to manipulate speech sounds on knowing alphabetic reading. Cognition, 24, 31-34.

Rodrigues, A., \& Pio da Rocha, J. M. (1994). Escala Colúmbia de Maturidade Intelectual. Manual. Rio de Janeiro: Centro Editor de Psicologia Aplicada.

Torgesen, J. K., Wagner, R. K., \& Rashotte, C. A . (1994). Longitudinal studies of phonological processing and reading. Journal of Learning Disabilities, 27(5), 276-286.

Magda Solange Vanzo Pestun, doutora em Ciências Médicas (Neurologia) pela Universidade de Campinas, é coordenadora do grupo de pesquisa "Desenvolvimento Humano" e professora no departamento de Psicologia Social e Institucional da Universidade Estadual de Londrina. Endereço para correspondência: Rodovia Celso Garcia Cid (445) Km. 380; Londrina, PR; CEP 86051-990. Tel.: (43) 3371-4487. E-mail: pestun@sercomtel.com.br 